\title{
Effects of virtual reality exercise for Korean adults with schizophrenia in a closed ward
}

\author{
Garam Jo' , Brenda Rossow-Kimball2, Gwitaek Park' ${ }^{1}$, Yongho Lee ${ }^{1, *}$ \\ 'Department of Physical Education, Seoul National University, Seoul, Korea \\ ${ }^{2}$ Faculty of Kinesiology and Heath Studies, University of Regina, Regina, SK, Canada
}

The purpose of this study was to examine the effects of virtual reality exercise (VRE) using Nintendo Wii-Fit on physical fitness of Korean adults with schizophrenia living in a mental health facility located in South Korea. Two male participants diagnosed with schizophrenia, ages 53 and 61, were recruited and selected for inclusion in this study. The intervention using the Nintendo Wii-Fit consisted of 35-min sessions, 3 times per week for 8 weeks and was facilitated by the primary researcher and two graduate students. The senior fitness test and $10-\mathrm{m}$ walking test were used to measure the physical functioning, specifically, physical fitness and mobility, of the participants. The study was divid- ed into three phases using an A-B-A single-subject design and involved multiple repeated measures of functional physical fitness. Both participants were evaluated each week for the duration of 18 weeks. Both participants exhibited measureable improvement in some of the physical fitness measures, but not in the mobility. These results thus provide preliminary evidence to support the use of VRE to improve physical function for Korean adults with schizophrenia as an alternative exercise regimen to the conventional exercise.

Keywords: Virtual reality exercise, Mental health, Schizophrenia

\section{INTRODUCTION}

Schizophrenia is one of the major mental health issues that may jeopardize health, well-being, and the overall quality of life of individuals (Ryu et al., 2015). According to the Diagnostic and Statistical Manual of Mental Disorders-5, "Schizophrenia is characterized by delusions, hallucinations, disorganized speech and behavior, and other symptoms that cause social or occupational dysfunction" (American Psychiatric Association, 2013). While studies attribute the primary causes of schizophrenia to biological or psychosocial factors, the major cause underlying the schizophrenia remain unclear (Carson et al., 2001). In addition to delusion and hallucination, schizophrenia often coexists with other health-related issues such as diabetes and hypertension (McGlashan, 1988). As a result, schizophrenia is one of the most costly disorders among adults (Bartels et al., 2003). De Hert et al. (2009) indicated that one of the main health issues in the management of schizophrenia is to reduce secondary physical health issues such as the risk of developing metabolic syndromes including diabetes, cardiovascular disease, hypertension, obesity, and hyperglycemia. Ryu et al. (2015) reported that individuals with schizophrenia do not generally engage in structured exercise on a regular basis and typically engage in other unhealthy lifestyles, such as having an irregular eating and sleeping schedule. They also reported that engaging in an exercise program with both cardiovascular and resistance training could help resolve physical health issues of individuals with schizophrenia by improving their cardiovascular strength and endurance, muscular strength, and flexibility. Thus, exercise can be part of a treatment protocol to meet the physical health needs of adults with schizophrenia. However, few studies have addressed the need for physical activity programs to help individuals with schizophrenia living in a restricted environment.

\section{Benefits of exercise}

Various treatments, including psychotherapy (Brus et al., 2012), pharmaceuticals, milieu therapy, and the use of exercise
${ }^{*}$ Corresponding author: Yongho Lee (iD https://orcid.org/0000-0002-6430-5327 Department of Physical Education, Seoul National University, Building 71-1, room 411, 1 Gwanak-ro, Gwanak-gu, Seoul 08826, Korea

Tel: +82-2-880-7762, Fax: +82-872-2867, E-mail: gophers@snu.ac.kr

Received: December 2, 2017 / Accepted: January 12, 2018
This is an Open Access article distributed under the terms of the Creative Commons Attribution Non-Commercial License (http://creativecommons.org/licenses/by-nc/4.0/) which permits unrestricted non-commercial use, distribution, and reproduction in any medium, provided the original work is properly cited. 
(Vancampfort et al., 2014), have been tested and administered to those with schizophrenia. The evidence of the benefits of exercise in general to improve physical, emotional, and cognitive function has been documented in the literature (Jeffs and Wiseman, 2013; Leenders et al., 2013; Madden, 2013). The benefits of exercise (e.g., positive effects on hypertension, body composition, cardiovascular endurance, emotional well-being, and cognitive function) for those with schizophrenia have also been well documented in the literature (Acil et al., 2008; Beebe et al., 2005; Doyne et al., 1983; Wu et al., 2007). In addition to its direct effects, participating in exercise with other people creates an environment in which participants feel emotionally stable and comfortable. This, in turn, facilitates social cohesion with other participants through nonverbal communication, thus helping to increase levels of physical capacity and opportunities for social networking (Zschucke et al., 2013).

Despite evidence of the positive effects of exercise, people with schizophrenia generally have low levels of physical fitness when compared to the general population because of the lack of opportunities to engage in meaningful physical activity (Faulkner et al., 2006). Adherence to exercise regimes is also a challenge for people with schizophrenia due to living in a restricted environment in Korea (i.e., a closed ward in a mental health facility) where opportunities to engage in meaningful exercise are not readily available (Lindamer et al., 2008). In addition, the side effects associated with schizophrenia medications including increased risk of metabolic syndrome, a decline in cognitive function, and cardiovascular disease can discourage individuals from engaging in physical activity (Akbostanci et al., 1999; De Hert et al., 2008; Hennekens et al., 2005; Jibson and Tandon, 1998). Particularly, individuals with schizophrenia tend to show symptoms of drowsiness, decreased attention span, weight gain, sexual dysfunction, decreased motor function and agitation due to medication side effects (Marder et al., 2004). Consequently, individuals with schizophrenia who live in a restricted environment at a psychiatric hospital may even be at greater risk for inactivity than the community-dwelling general population.

\section{Virtual reality, ecological task analysis, and affordance Virtual reality and ecological task analysis}

Virtual reality (VR), also referred to as immersive multimedia or computer-simulated life, involves replicating an environment to simulate physical presence in real or imagined worlds, and allows the user to interact in that world. VR can be theoretically supported for use as an intervention, as it involves ecological task analysis (ETA), which is grounded in the concept of "affordance"
(Hahn and Lee, 2001). ETA initially presented by Davis and Burton (1991), is probably a detailed and comprehensive model that applies dynamical systems and related theories to the assessment and intervention of movement development (Burton and Davis, 1996). ETA involves a movement system that appears because of the interaction among task performers, task goals, and the environment. It is composed of four steps: (a) setting up the task goals by structuring the physical and social environments; (b) enabling choices of movement solutions; (c) manipulating performer, environmental, or task variable; and (d) providing instruction (Davis and Burton, 1991). Through these steps, individuals can improve functional movement pattern as they gain control over his or her surrounding environment. These steps are based on the concept of affordance described as follows.

\section{Affordance}

Affordance refers to situations during which individuals exert their highest performance when they feel their surrounding environments are most accommodating (Davis and Burton, 1991). For example, a playground affords for playing while it does not afford for studying because of noise generated from the surrounding environment. While most individuals may feel an environment affording for interaction between the individuals and the environment, some individuals may have a personal constraint that hinders effective interaction with an environment that may be accessible to others. In that case, the environment needs to be modified to allow the individuals to feel afforded. However, not all environments allow for modification. VR permits environmental modification to afford for effective interaction between individuals and environments. In the case of patients diagnosed with schizophrenia living in a restricted environment, participating in various forms of exercise with other people is difficult because of the restricted environment in a closed ward (Lindamer et al., 2008). Virtual reality exercise (VRE) can provide an affordable means to directly experience various forms of exercise that are not easily experienced in reality.

VRE can encourage physical activity at anytime, anywhere and can facilitate compliance with regular physical activity (de Bruin et al., 2010). Programs based on VR can safely and easily simulate a real environment to accommodate individuals with unique needs. It also offers a cost-effective intervention while stimulating both physical and cognitive skills in a socially interactive setting (Weybright et al., 2010). This motivates participants to not only engage in physical activity but also encourages them to participate in the program for a sustained period of time because it is en- 
joyable, fun, and safe (Flynn et al., 2007). VRE is beneficial in that they promote improved voluntary movement control and coordination for individuals with cerebral palsy (Bryanton et al., 2006), motor control among those with Parkinson's disease (Ferrarin et al., 2004), and mobility among those who have experienced cerebrovascular accidents (Jaffe et al., 2004). However, few studies have been conducted to test the use of VRE for patients diagnosed with schizophrenia, who reside in a restricted living environment, and who lack opportunities to engage in physical activities. VRE can provide this population with an accessible environment for their physical activity needs.

The main purpose of the current study was to examine the effects of VRE using Nintendo Wii-Fit (Nintendo of Korea, Seoul, Korea) widely used software in VRE on the physical functioning capacity of Korean adults with schizophrenia living in a restricted environment in a mental health facility. An additional purpose was to examine whether VRE can be an effective, safe, and enjoyable alternative regimen to traditional exercise programs that ultimately increases adherence to exercise for those living in a restricted environment.

\section{MATERIALS AND METHODS}

\section{Participants}

Participants for this study were recruited from a psychiatric hospital located in South Korea. Participants were included if they were: (a) 50 or older in age, (b) 25-year postschizophrenia diagnosis, (c) independent in activities of daily living, and (d) able to participate in physical activity requiring musculoskeletal movement without difficulty as determined by self-report Fifteen initial participants were recruited through a poster advertisement and through personal contacts at the hospital. Only four patients satisfied the inclusion criteria and were selected in the study. However, due to health issues that occurred after the selection process, two of the participants had to withdraw during the initial phase of the study, leaving only 2 participants. One patient, Kim, is a 61-year-old male patient who was diagnosed as having schizophrenia in 1986. The other patient, Park, is a 53-year-old male patient who was diagnosed as having schizophrenia in 1980. Pseudonyms are used to protect anonymity of study participants.

\section{Setting}

This study was performed in a psychiatric hospital located in the mid-region of South Korea. The hospital did not provide structured exercise on a regular basis to patients, so the partici- pants in this study had not engaged in structured exercise in the hospital before taking part in the current research. The VRE program was facilitated by primary researcher and two graduate students studying at the affiliated school assisted the primary researcher.

The study was approved by the Institutional Review Board at the primary researcher's affiliated university for the use of human participants in research. A consent form was distributed and read to the participants.

\section{Research design}

The current study followed a single-subject research design that is appropriate for small sample sizes and multiple repeated measures over a period of time (Dattilo et al., 2000). In single-subject research, changes in a participant's behavior are plotted on a graph and analysis is done visually without the use of inferential statistical analysis (Kim and Kim, 2009). For this study, an A-B-A, labeled as "A1-B-A2," single subject research design was used and administered in three phases: baseline (A1), intervention (B), and final baseline (A2). Individual data were collected and assessed during the A1 phase. The VRE program was implemented during the $\mathrm{B}$ phase, and changes were, again, observed and recorded during the A2 phase. When using this design, changes are expected during and shortly after the intervention as effects, and no change or regression to $\mathrm{A} 1$ is expected when no intervention is provided during the A2 phase (Retarekar et al., 2009). Although A-B-A-B design is conventionally used in single subject design research, A-B-A design still can provide confidence in the researcher's ability to determine the impact of treatment (Zhan and Ottenbacher, 2001). Retarekar et al. (2009) used this design as their research approach to investigate the effects of an exercise program for a child with cerebral palsy, and reported that the use of this research procedure can provide valuable clinical information due to its feasibility in a clinical setting when used with the small number of participants in a limited time frame.

Research was conducted for a duration of 18 weeks for each participant. Each of the A1 and A2 phases consisted of a 3-week assessment without an intervention while the B phase lasted 8 weeks with the intervention. All measurements were assessed 14 times during the course of the study ( 3 times for A1, 8 times for $\mathrm{B}, 3$ times for A2). All assessments were administered and facilitated by the primary researcher and two graduate students.

\section{Dependent variables and assessment instruments}

The four variables of physical fitness, mobility, reaction time, and body composition were measured to assess the participants' 
physical functioning, as is generally recommended when assessing the physical function of adults with schizophrenia (Birkett et al., 2007; Gale and Holzman, 2000; Ryu et al., 2015). However, because the latter two variables were only measured 4 times throughout the course of the study, the data were not included in the final analysis. The senior fitness test (SFT) for physical fitness, and a 10-m walking test for mobility were used to examine the effects of the VRE on physical functional capacity. Definitions and detailed information on the assessment tools is described as follows.

\section{Physical fitness}

Physical fitness is defined as "the ability to carry out daily tasks with vigor and alertness, without undue fatigue and with ample energy to enjoy leisure-time pursuits and to meet unforeseen emergencies" (Caspersen et al., 1985). Low physical fitness has been recognized as a prominent health related risk factor for people with schizophrenia for cardiovascular diseases and metabolic syndrome (Vancampfort et al., 2014). The SFT, which was developed by Rikli and Jones (2013), assesses the physical fitness of older adults by focusing on the ability to perform basic functional movements, such as walking, stair climbing, and standing up. It also assesses six areas of fitness in seven different test modes. The test is safe and requires no special equipment to measure the physiological nature of fitness. Six areas of functional fitness are measured by performing the following movement tasks: chair stand (lower body strength), arm curl (upper body strength), 2-min step (cardiovascular endurance), chair sit and reach (lower body flexibility), back stretch (upper body flexibility), and 8 foot up and go (agility/dynamic balance). The SFT reports reliability with Cronbach $\alpha$ of 0.89 for the chair stand, 0.81 for the arm curl, 0.90 for the 2-min step, 0.95 for sit and reach, 0.96 for back stretch, and 0.95 to the 8 foot up and go. This test also reports a validity of 0.77 for the chair stand, 0.74 for the 2 -min step, and 0.83 for sit and reach. The tests were administered in accordance with SFT manual by two trained graduate research assistants. More detailed information about the test is well described in SFT by Rikli and Jones (2013).

\section{Mobility (walking ability)}

Patients with schizophrenia frequently show disabling gait deficits such as decreased walking speed, reduced stride length and balance deficits (Putzhammer et al., 2004). A 10-m walking test was conducted to assess participants' mobility. The purpose of this test is to assess walking speed in meters per second over a short distance. A 14-m walking test was conducted to control for the acceleration and deceleration at the beginning and at the end of the walking test, but only the time to walk $10 \mathrm{~m}$ in distance was measured. Liston and Brouwer (1996) reported that the 10-m walking test was reliable, with a Cronbach a of 0.96 .

\section{Data collection procedures}

The interventions and assessments were conducted consistently at the same time of day in the morning before providing the intervention. However, all tests were not conducted following the same order in an effort to prevent potential practice effect. Three steps were used to develop the intervention protocol. First, a thorough review of the literature related to VRE programs and an activity analysis of protocols used in previous VRE studies were conducted to identify and analyze essential components to be included in the protocol. Based on our analysis, the intervention of this study included components to stimulate cardiovascular endurance, muscular strength, agility, and attentional control. After our initial protocol was developed, an expert panel was consulted to further discuss validity of the protocol for the given population. Three experts in exercise science were involved in the process. Finally, a pilot test was conducted for adults enrolled at another exercise program offered at the researcher's affiliated school to ensure the trustworthiness of the protocol.

\section{A1 phase}

The A1 phase lasted three weeks and worked to establish the baseline data of the participants prior to commencing the intervention phase (B). The participants completed all assessments prior to starting the intervention during the A1 phase. The SFT and the 10-m walking test were conducted three times for 3 weeks. No structured exercise program was carried out at the hospital during the A1 phase. Participants received their routine medical treatment at this time.

\section{Bphase}

The VRE protocol was developed and administered to the participants using the Nintendo Wii-Fit software. Nintendo Wii-Fit is extensively used in rehabilitation settings as a VRE program for people who cannot actively participate in real environment exercise because of various physical limitations (Pompeu et al., 2012). The Wii-Fit program is equipped with a balance board that detects participants' movement and transmits participants' movement data to the Wii console. Participants engaged in a VRE program 3 times per week for 35 min over an 8 -week period together at the same time. During the B phase, SFT and the 10-m walking 
test were administered every week, totaling 8 times, while body composition and reaction time were measured twice at halfway and the end of the intervention.

Each VRE program consisted of 5 min of stretching, 25 min of VRE, and 5 min of stretching at the end of the program. As in the $\mathrm{A} 1$ and $\mathrm{A} 2$ phases, no other exercise or physical activity programs were administered to the participants. The participants then completed all of the intervention sessions during the $\mathrm{B}$ phase. See Table 1 for the specific VRE exercise protocol.

\section{A2 phase}

As in the A1 phase, all of the tests were administered to the participants to establish baseline measure on dependent variables in the absence of the intervention. The tests were performed one month after the intervention to clear the effects of the intervention. The $10-\mathrm{m}$ walking test was conducted 3 times during this phase. The body composition and reaction time were assessed once at the beginning of this phase.

\section{Data analysis}

Analysis was based on visual interpretation of plotted raw data. The most common data-analysis approach used in single-subject designs is visual analysis (Haegele and Hodge, 2015). By using this approach, researchers can indicate evidence of a causal relation between an intervention and outcome variable (Cooper et al., 2007; Kratochwill et al., 2010). In this study, dependent variables (i.e., physical fitness, mobility) for each participant were plotted on a graph to show trend lines. Trend line was established through changes in slope and extent within each plot. Because of differences in the frequency of data collected at each stage of the study (baseline, intervention, post intervention baseline) and the small number of measurements, statistical analysis to support the visual interpretation of the data was not appropriate but slope and level changes are appropriate for small number of research participants. The typical analysis includes trend, level of change, and slope. Therefore, the average rating for each participant was used during each stage for physical fitness and mobility.

\section{RESULTS}

The current study implemented VRE as a therapeutic modality to the individuals with schizophrenia living in a closed ward in a psychiatric hospital in South Korea. Two Korean adults with schizophrenia were selected and included in the current study. Some positive changes were observed for both participants during
Table 1. Exercise protocol

\begin{tabular}{ccl}
\hline Time $(\mathrm{min})$ & Type of exercise & The contents of exercise \\
\hline 5 & & Warm up \\
10 & Strength training & Lunge \\
& & Squat \\
15 & Balance and cardiovascular & Ski jump \\
& endurance exercise & Ski slalom \\
& & Penguin seesaw \\
& & Table tilt \\
& & Wire walking \\
& & Cool down \\
\end{tabular}

and after the interventions. There were positive changes in terms of the upper body strength, upper and lower body flexibility, cardiovascular endurance, and agility/dynamic balance in the SFT as well as a slight improvement in mobility in the 10 -m walking test. Little to no desired changes were observed in lower body strength in the SFT. Further details on the changes as a result of the intervention are described as follows.

\section{Physical fitness}

Physical fitness was measured by the SFT that included measures of the arm curl, back stretch, chair sit and reach, 2-min step test, 8 foot up and go, and 10-m walking test.

\section{Arm curl (upper body strength)}

For upper body strength in the arm curl test, Kim had a decelerating trend and Park had a stable trend in A1. However, Kim and Park had a slightly accelerating trend with the exercise during the $\mathrm{B}$ phase. Kim demonstrated an improvement from an average score of 13 during the $\mathrm{A} 1$ phase to 13.5 during the $\mathrm{B}$ phase and then increased again in the A2 phase to 15. Park scored an average of 19 during the $\mathrm{A} 1$ phase in the arm curl and then scored an average of 19.3 during the B phase, which is slight increase from the A1 phase. In the follow-up, he demonstrated a decrease to an average score of 18 , which was a change that was indicative of an intervention effect (Table 2, Fig. 1)

\section{Back stretch (upper body flexibility)}

For upper body flexibility in the back stretch test, both Kim and Park showed an improvement. Kim had a decelerating trend and Park had a slightly accelerating trend in the A1. After that, Kim had slightly increasing trend and Park had sharply accelerating trend in the B phase. Park's trend sharply decreased and Kim maintained increasing trend in the $\mathrm{A} 2$ phase as indicated in the Table 3 and Fig. 2. 
Table 2. Arm curl

\begin{tabular}{lccc}
\hline Patient & A1 & B & A2 \\
\hline Kim & $13 \pm 1.0$ & $13.5 \pm 0.9$ & $15 \pm 2.1$ \\
Park & $19 \pm 1.7$ & $19.3 \pm 0.9$ & $18 \pm 2.0$ \\
\hline
\end{tabular}

Values are presented as mean \pm standard deviation.

$A 1$, baseline; $B$, intervention; $A 2$, final baseline.

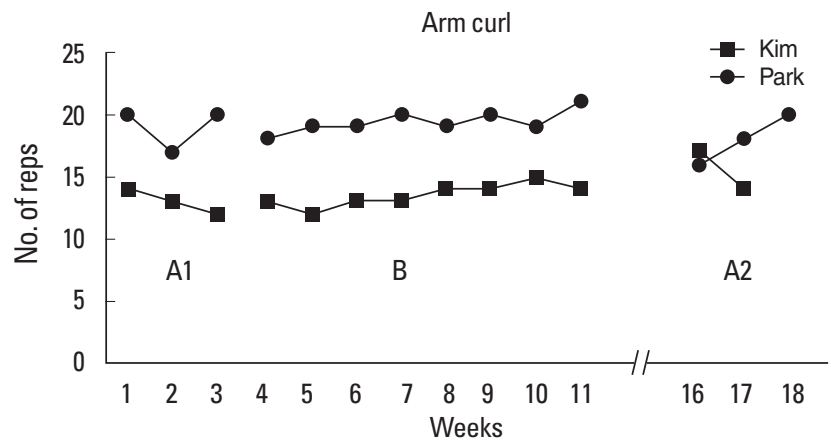

Fig. 1. Data trend line of participants for arm curl. A1, baseline; B, intervention; A2, final baseline.

\section{Chair sit and reach (lower flexibility)}

For lower flexibility in the chair sit and reach test, Kim had a stable trend and Park had a decelerating trend in the A1 phase. However, Kim and Park had sharply accelerating trend in the B1 phase. Park maintained increasing trend and Kim had decreasing trend in the A2 phase. As shown in the Table 4 and Fig. 3, Kim improved from an average score of 1.6 during the A1 phase to an average score of 5.5 during the B phase. An improvement was continually observed in the A2 phase for Kim, and Park also showed an increase in terms of the lower flexibility during the $\mathrm{B}$ phase. In the follow up at the A2 phase in which decreasing trend was expected to indicate an intervention effect, he scored an average of 15 , which is an increase from an average of 9.5 during the $B$ phase.

\section{Two-minute step test (endurance)}

Table 5 and Fig. 4 display an increasing trend in cardiovascular endurance for both participants in the 2-min step test. After a stable A1 trend, both exhibited an improvement in the B phase and then a decline in the A2 phase. Kim scored an average of 62.6 during the A1 phase. As he participated in the intervention, an increase in trend was observed to a score of 93 at average during the $\mathrm{B}$ phase. In the absence of an intervention during the A2 phase, the score decreased. In the meantime, Park, similarly, showed a trend for similar changes as those for Kim. Park had an increasing trend as he scored an average of 73.6 during the A1
Table 3. Back stretch

\begin{tabular}{lccc}
\hline Patient & A1 & B & A2 \\
\hline Kim & $-39.6 \pm 4.9$ & $-38.6 \pm 2.3$ & $-26.5 \pm 2.1$ \\
Park & $-27.0 \pm 4.7$ & $-3.25 \pm 19.0$ & $-19 \pm 3.6$ \\
\hline
\end{tabular}

Values are presented as mean \pm standard deviation. $A 1$, baseline; $B$, intervention; $A 2$, final baseline.

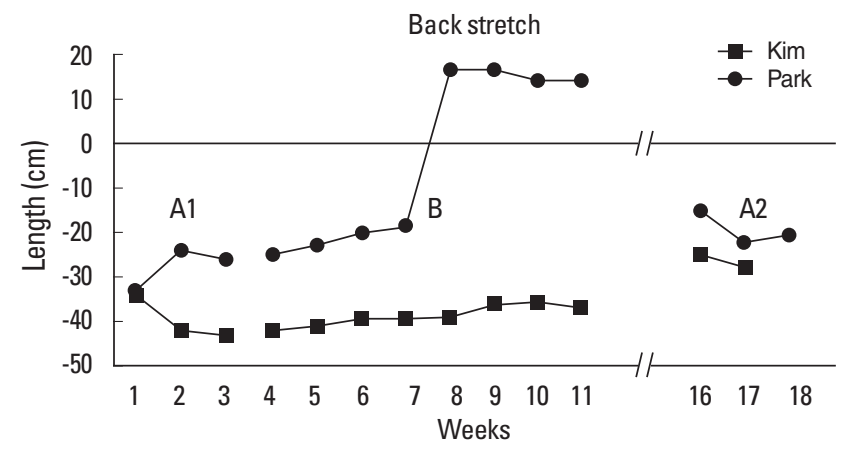

Fig. 2. Data trend line of participants for back stretch. $A 1$, baseline; $B$, intervention; $A 2$, final baseline.

Table 4. Chair sit and reach

\begin{tabular}{lccr}
\hline Patient & A1 & B & \multicolumn{1}{c}{ A2 } \\
\hline Kim & $1.6 \pm 0.5$ & $5.5 \pm 2.4$ & $6.0 \pm 1.4$ \\
Park & $4.6 \pm 2.08$ & $9.5 \pm 2.3$ & $15.0 \pm 0.8$ \\
\hline
\end{tabular}

Values are presented as mean \pm standard deviation. $A 1$, baseline; $B$, intervention; $A 2$, final baseline.

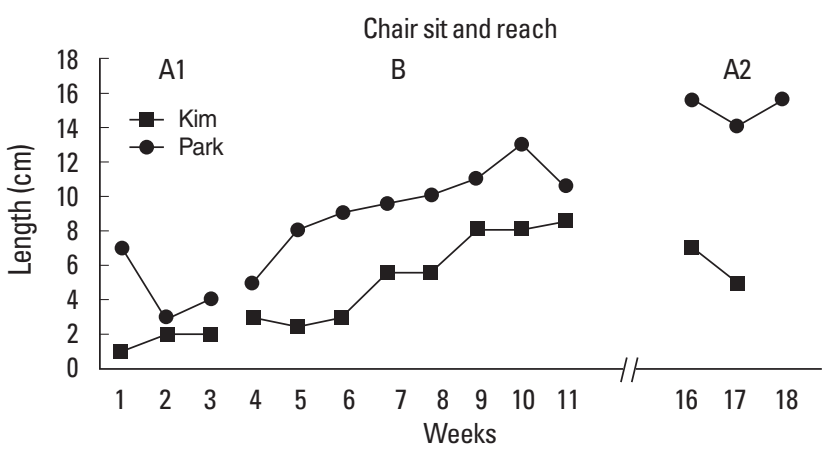

Fig. 3. Data trend line of participants for chair sit and reach. A1, baseline; $B$, intervention; $A 2$, final baseline.

phase and then improved to 98.8 on average during the B phase. A decreasing trend was observed during the A2 phase in the follow up, indicating an effect of VRE on cardiovascular endurance.

\section{Foot up and go (agility/dynamic balance)}

As shown in Table 6, there is little change in the average score in terms of agility/dynamic balance for both participants. Howev- 
Table 5. Two-min step test

\begin{tabular}{lccc}
\hline Patient & A1 & \multicolumn{1}{c}{ B } & A2 \\
Kim & $62.6 \pm 0.5$ & $93.8 \pm 8.3$ & $78.0 \pm 25.4$ \\
Park & $73.6 \pm 2.3$ & $98.8 \pm 13.5$ & $100.6 \pm 2.5$
\end{tabular}

Values are presented as mean \pm standard deviation.

$A 1$, baseline; $B$, intervention; $A 2$, final baseline.

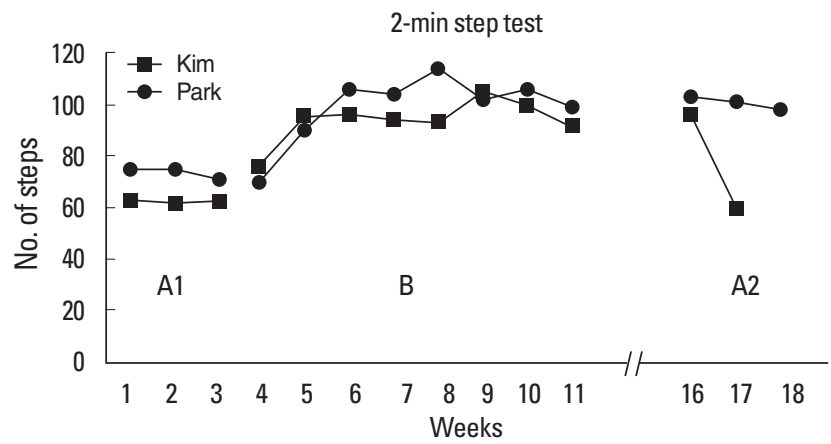

Fig. 4. Data trend line of participants for 2-min step test. A1, baseline; B, intervention; $A 2$, final baseline.

er, Fig. 5 displays a slight decrease in trend in the graphic display for Kim and an increase in trend during the A2 phase, indicating an effect of the VRE intervention on agility/dynamic balance. Meanwhile, Kim showed little changes in the graphic display in Fig. 5.

\section{Mobility}

The 10-m walk test was conducted to assess participants' changes in mobility. Table 7 and Fig. 6 show the changes in mobility of the participants. Neither participant showed a change in the average scores for the 10-m walk test, as shown in Table 7. Although the average score does not show changes, Fig. 6 indicates changes for both Kim and Park. During the A1 phase, Kim showed an increasing trend in the time consumed for the $10-\mathrm{m}$ walk. Then, he showed an increase in performance level as indicated by a decrease in time for the 10-m walk test. Finally, during the A2 phase, although slight, Kim showed a decrease in trend in time spent on the 10-m walk test.

Fig. 6 also shows positive changes in Park in the time spent for walking 10-m. As Park participated in the intervention, the time spent walking 10-m decreased from the 6th week and started to increase in week 9 during the B phase. After the intervention, a decrease was observed in the time to walk $10 \mathrm{~m}$ suggesting the intervention was effective.
Table 6. Eight foot up and go

\begin{tabular}{lcrr}
\hline Patient & A1 & B & \multicolumn{1}{c}{ A2 } \\
\hline Kim & $10.6 \pm 0.11$ & $10.3 \pm 0.3$ & $10.2 \pm 0.7$ \\
Park & $8.3 \pm 0.0$ & $8.3 \pm 0.6$ & $8.5 \pm 0.3$ \\
\hline
\end{tabular}

Values are presented as mean \pm standard deviation.

$A 1$, baseline; $B$, intervention; $A 2$, final baseline.

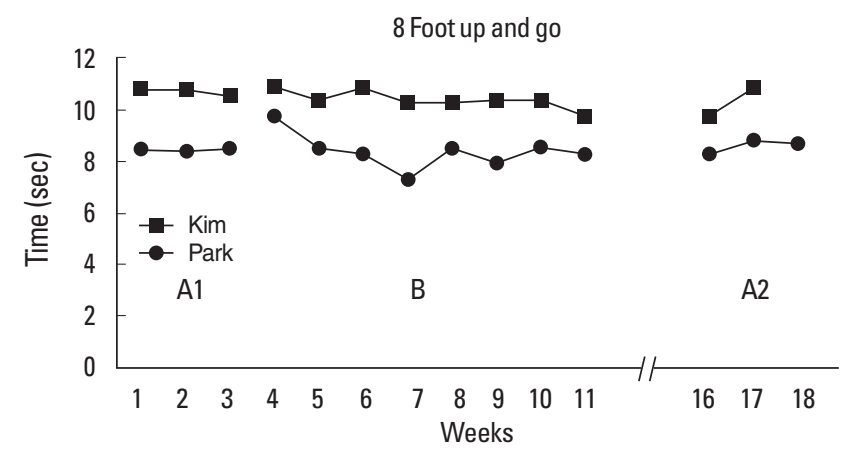

Fig. 5. Data trend line of participants for 8 foot up and go. A1, baseline; $B$, intervention; $A 2$, final baseline.

Table 7. Ten-meter walking test

\begin{tabular}{lrrr}
\hline Patient & A1 & B & \multicolumn{1}{c}{ A2 } \\
\hline Kim & $11.4 \pm 0.8$ & $11.3 \pm 0.5$ & $12.4 \pm 0.4$ \\
Park & $8.5 \pm 0.3$ & $8.8 \pm 0.5$ & $8.6 \pm 0.3$ \\
\hline
\end{tabular}

Values are presented as mean \pm standard deviation.

$A 1$, baseline; $B$, intervention; $A 2$, final baseline.

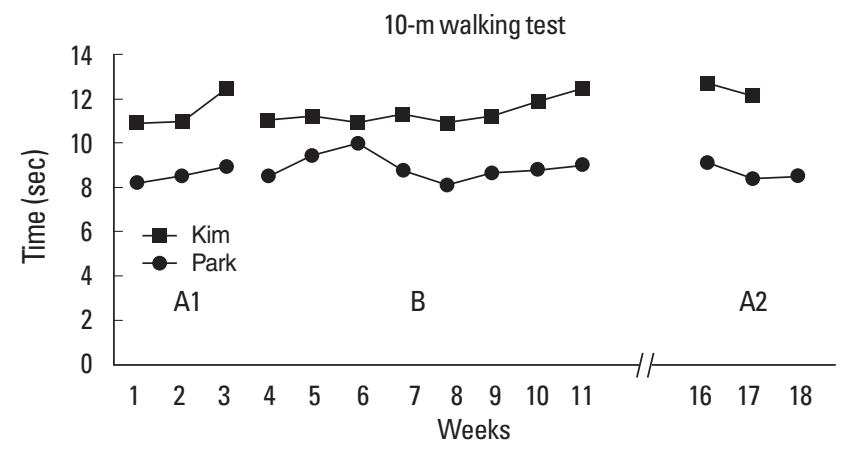

Fig. 6. Data trend line of participants for 10-m walking test. A1, baseline; $B$, intervention; $A 2$, final baseline.

\section{DISCUSSION}

This study examined the effects of a VRE intervention on the physical functioning (physical fitness, mobility) of two research participants with schizophrenia in Korea. Although the results are not generalizable due to the nature of a single subject design that only provides insight to a given context, the intervention yielded 
some positive effects on physical fitness; however, changes in upper and lower body strength were not clearly observed. As the results indicate, there were some positive changes in the physical fitness of the participants at the B phases of the study. However, only three measures had baseline trends that allow us to conclude that the intervention caused the improved scores. These measures included the 2-min step, 8 foot up and go, and 10-m walk test. The findings indicate that moderate participation in VRE can produce a positive impact on physical fitness for those living in a restricted environment. The participants exhibited the most improvement in cardiovascular endurance in the 2-min step test in this study. Even after the program concluded, they maintained an improved level of cardiovascular endurance although it eventually declined in the absence of exercise. This may be due to the increased motivation of the participants to participate in physical activity since the improvement in the A2 phase was found in some other variables. Research suggests that adherence to exercise can increase when the exercise is conducted in a safe and pleasurable environment (Lee and Kim, 2013). However, it is premature to conclude that the improvement in the $\mathrm{A} 2$ phase could be attributed to the increase in physical activity since the current study did not assess the physical activity level of the participants.

VRE has been shown to have positive effects on balance and gait in the general public (Song et al., 2009). This is due in part to the improved lower body strength. Thus, the researchers of this study expected that participants should improve their balance as a result of the program. However, an improvement in balance as measured by 8 foot up and go test was not clearly observed at the expected level even though a slight improvement was observed. This may be due to the nature of research participants' diagnostic condition. The participants in this study were diagnosed with schizophrenia, while previous studies have tested the effects of VRE on balance for people without any disabilities. At times, the participants were confused and disoriented during the exercise due, in part, to the medication they were taking and this could have also resulted in a lack of positive effects on balance after the intervention.

As one's surrounding environment is one of the factors known to influence human movement performance based on the concept of affordance, VR can provide a surrounding environment that is more accommodating than a real environment experience to individuals who live in restricted living environments. As one's environment becomes more accommodating thereby affording for optimal performance, individuals can manage tasks more effectively. VRE may provide an environment that is more accommodating and less challenging while still providing similar effects to that of a real environment experience for those living in a restricted living environment such as mental health facility. It is cost-effective intervention requiring both physical and cognitive skills yet still promoting social interaction in an interactive environment (Weybright et al., 2010). It can also be used outside the treatment setting. Not only can it provide additional opportunity to improve functional capacity outside the treatment setting, but also facilitate positive social interaction with family and friends (Kenuk et al., 2015). Finally, as VRE programs are further developed technologically, they might serve as a form of exercise used to stimulate real environments in restricted living experiences. Therefore, based on results of the current study, VRE can be added to the small number of tested exercise intervention protocols as effective and efficient therapeutic regime in mental health care setting.

\section{CONFLICT OF INTEREST}

No potential conflict of interest relevant to this article was reported.

\section{REFERENCES}

Acil AA, Dogan S, Dogan O. The effects of physical exercises to mental state and quality of life in patients with schizophrenia. J Psychiatr Ment Health Nurs 2008;15:808-815.

Akbostanci MC, Atbaşoğlu EC, Balaban H. Tardive dyskinesia, mild druginduced dyskinesia, and drug-induced parkinsonism: risk factors and topographic distribution. Acta Neurol Belg 1999;99:176-181.

American Psychiatric Association. Diagnostic and statistical manual of mental disorders (DSM-5). Washington, DC: American Psychiatric Publishing; 2013.

Bartels SJ, Clark RE, Peacock WJ, Dums AR, Pratt SI. Medicare and medicaid costs for schizophrenia patients by age cohort compared with costs for depression, dementia, and medically ill patients. Am J Geriatr Psychiatry 2003;11:648-657.

Beebe LH, Tian L, Morris N, Goodwin A, Allen SS, Kuldau J. Effects of exercise on mental and physical health parameters of persons with schizophrenia. Issues Ment Health Nurs 2005;26:661-676.

Birkett P, Sigmundsson T, Sharma T, Toulopoulou T, Griffiths TD, Reveley A, Murray R. Reaction time and sustained attention in schizophrenia and its genetic predisposition. Schizophr Res 2007;95:76-85.

Brus M, Novakovic V, Friedberg A. Psychotherapy for schizophrenia: a review of modalities and their evidence base. Psychodyn Psychiatry 2012;40:609-616. 
Bryanton C, Bossé J, Brien M, McLean J, McCormick A, Sveistrup H. Feasibility, motivation, and selective motor control: virtual reality compared to conventional home exercise in children with cerebral palsy. Cyberpsychol Behav 2006;9:123-128.

Burton AW, Davis WE. Ecological task analysis utilizing intrinsic measures in research and practice. Hum Mov Sci 1996;15:285-314.

Carson RC, Butcher JN, Mineka S. Abnormal psychology and modern life. Boston (MA): Allyn \& Bacon; 2001.

Caspersen CJ, Powell KE, Christenson GM. Physical activity, exercise, and physical fitness: definitions and distinctions for health-related research. Public Health Rep 1985;100:126-131.

Cooper JO, Heron TE, Heward WL. Applied behavior analysis. 2nd ed. Upper Saddle River (NJ): Pearson/Merrill-Prentice Hall; 2007.

Dattilo J, Gast DL, Loy DP, Malley S. Use of single-subject research designs in therapeutic recreation. Ther Recreat J 2000;34:253-270.

Davis WE, Burton AW. Ecological task analysis: translating movement behaviour into practice. Adapt Phys Act Q 1991;8:154-177.

de Bruin ED, Schoene D, Pichierri G, Smith ST. Use of virtual reality technique for the training of motor control in the elderly. Some theoretical considerations. Z Gerontol Geriatr 2010;43:229-234.

De Hert M, Schreurs V, Sweers K, Van Eyck D, Hanssens L, Sinko S, Wampers M, Scheen A, Peuskens J, van Winkel R. Typical and atypical antipsychotics differentially affect long-term incidence rates of the metabolic syndrome in first-episode patients with schizophrenia: a retrospective chart review. Schizophr Res 2008;101:295-303.

De Hert M, Schreurs V, Vancampfort D, VAN Winkel R. Metabolic syndrome in people with schizophrenia: a review. World Psychiatry 2009; 8:15-22.

Doyne EJ, Chambless DL, Beutler LE. Aerobic exercise as a treatment for depression in women. Behav Ther 1983;14:434-440.

Faulkner G, Cohn T, Remington G. Validation of a physical activity assessment tool for individuals with schizophrenia. Schizophr Res 2006; 82:225-231.

Ferrarin M, Brambilla M, Garavello L, Di Candia A, Pedotti A, Rabuffetti M. Microprocessor-controlled optical stimulating device to improve the gait of patients with Parkinson's disease. Med Biol Eng Comput 2004;42:328-332.

Flynn S, Palma P, Bender A. Feasibility of using the Sony PlayStation 2 gaming platform for an individual poststroke: a case report. J Neurol Phys Ther 2007;31:180-189.

Gale HJ, Holzman PS. A new look at reaction time in schizophrenia. Schizophr Res 2000;46:149-165.

Haegele JA, Hodge SR. The applied behavior analysis research paradigm and single-subject designs in adapted physical activity research. Adapt Phys Activ Q 2015;32:285-301.
Hahn JS, Lee KS. A theoretical review on designing virtual reality in the teaching-learning process. J Educ Techno 2001;17:133-163.

Hennekens CH, Hennekens AR, Hollar D, Casey DE. Schizophrenia and increased risks of cardiovascular disease. Am Heart J 2005;150:11151121.

Jaffe DL, Brown DA, Pierson-Carey CD, Buckley EL, Lew HL. Stepping over obstacles to improve walking in individuals with poststroke hemiplegia. J Rehabil Res Dev 2004;41(3A):283-292.

Jeffs E, Wiseman T. Randomised controlled trial to determine the benefit of daily home-based exercise in addition to self-care in the management of breast cancer-related lymphoedema: a feasibility study. Support Care Cancer 2013;21:1013-1023.

Jibson MD, Tandon R. New atypical antipsychotic medications. J Psychiatr Res 1998;32:215-228.

Kenuk S, Borders R, Palmer K, Nelson, R. Using the Wii for functional improvements in individuals with brain injuries. Ther Recreat J 2015; 49:261-264.

Kim NJ, Kim YW. Research methods in education. Seoul: Chung Mock; 2009.

Kratochwill TR, Hitchcock J, Horner RH, Levin JR, Odom SL, Rindskopf DM, Shadish WR. Single-case designs technical documentation. Washington, DC: Institute of Education Sciences; 2010 [cited 2017 Jul 25]. Available from: https://ies.ed.gov/ncee/wwc/Document/229.

Lee YH, Kim KT. Tai Chi as fall prevention intervention: an in-depth review of randomized controlled trials and suggestion to Taekwondo. J Kinesiol 2013;15:1-11.

Leenders M, Verdijk LB, van der Hoeven L, van Kranenburg J, Nilwik R, van Loon LJ. Elderly men and women benefit equally from prolonged resistance-type exercise training. J Gerontol A Biol Sci Med Sci 2013;68: 769-779.

Lindamer LA, McKibbin C, Norman GJ, Jordan L, Harrison K, Abeyesinhe S, Patrick K. Assessment of physical activity in middle-aged and older adults with schizophrenia. Schizophr Res 2008;104:294-301.

Liston RA, Brouwer BJ. Reliability and validity of measures obtained from stroke patients using the Balance Master. Arch Phys Med Rehabil 1996;77:425-430.

Madden KM. Evidence for the benefit of exercise therapy in patients with type 2 diabetes. Diabetes Metab Syndr Obes 2013;6:233-239.

Marder SR, Essock SM, Miller AL, Buchanan RW, Casey DE, Davis JM, Kane JM, Lieberman JA, Schooler NR, Covell N, Stroup S, Weissman EM, Wirshing DA, Hall CS, Pogach L, Pi-Sunyer X, Bigger JT Jr, Friedman A, Kleinberg D, Yevich SJ, Davis B, Shon S. Physical health monitoring of patients with schizophrenia. Am J Psychiatry 2004;161:13341349.

McGlashan TH. A selective review of recent North American long-term 
followup studies of schizophrenia. Schizophr Bull 1988;14:515-542.

Pompeu JE, Mendes FA, Silva KG, Lobo AM, Oliveira Tde P, Zomignani $\mathrm{AP}$, Piemonte ME. Effect of Nintendo Wii ${ }^{\mathrm{TM}}$-based motor and cognitive training on activities of daily living in patients with Parkinson's disease: a randomised clinical trial. Physiotherapy 2012;98:196-204.

Putzhammer A, Heindl B, Broll K, Pfeiff L, Perfahl M, Hajak G. Spatial and temporal parameters of gait disturbances in schizophrenic patients. Schizophr Res 2004;69:159-166.

Retarekar R, Fragala-Pinkham MA, Townsend EL. Effects of aquatic aerobic exercise for a child with cerebral palsy: single-subject design. Pediatr Phys Ther 2009;21:336-344.

Rikli RE, Jones CJ. Senior fitness test manual. Champaign (IL): Human Kinetics; 2013.

Ryu SH, Song BK, Kim YS, Kown HJ. The effect of combined exercise on physical fitness and depression symptom in patients with schizophrenia. J Adapt Phys Actn Exerc 2015;23:39-48.

Song CH, Shin WS, Lee KJ, Lee SW. The effect of a virtual reality-based exercise program using a video game on the muscle strength, balance and gait abilities in the elderly. J Korean Geriatr Soc 2009;29:12611275.

Vancampfort D, Probst M, De Hert M, Soundy A, Stubbs B, Stroobants M, De Herdt A. Neurobiological effects of physical exercise in schizophrenia: a systematic review. Disabil Rehabil 2014;36:1749-1754.

Weybright EH, Dattilo J, Rusch F. Effects of an interactive video game (Nintendo Wii) on older women with mild cognitive inpairment. Ther Recreat J 2010;44:271-287

Wu MK, Wang CK, Bai YM, Huang CY, Lee SD. Outcomes of obese, clozapine-treated inpatients with schizophrenia placed on a six-month diet and physical activity program. Psychiatr Serv 2007;58:544-550.

Zhan S, Ottenbacher KJ. Single subject research designs for disability research. Disabil Rehabil 2001;23:1-8.

Zschucke E, Gaudlitz K, Ströhle A. Exercise and physical activity in mental disorders: clinical and experimental evidence. J Prev Med Public Health 2013;46 Suppl 1:S12-S21. 